\title{
A Summary of the Research on the Teaching Mode of MOOCs
}

\author{
Hong Zhao \\ School of Public Administration and Emergency Management, Jinan University, Guangzhou, China \\ Email: zhaohong_jnu@126.com
}

How to cite this paper: Zhao, H. (2019) A Summary of the Research on the Teaching Mode of MOOCs. Open Journal of Social Sciences, 7, 96-109.

https://doi.org/10.4236/jss.2019.72007

Received: December 19, 2018

Accepted: February 10, 2019

Published: February 13, 2019

Copyright $\odot 2019$ by author(s) and Scientific Research Publishing Inc. This work is licensed under the Creative Commons Attribution International License (CC BY 4.0).

http://creativecommons.org/licenses/by/4.0/

\begin{abstract}
MOOCs have become a new type of teaching mode, which has attracted wide attention of researchers and formed rich research results. This paper summarizes the characteristics of the teaching mode of MOOCs, the interaction between "teaching" and "study" in the teaching mode of MOOCs, the influence of the teaching mode of MOOCs on traditional teaching, the advantages and disadvantages of the teaching mode of MOOCs and the improvement strategy, in order to deepen the understanding of the study of the MOOCs teaching model. Through the review, the article believes that there are three shortcomings in the current research on MOOCs: the lack of research on the improvement strategy of MOOCs, the lack of empirical analysis on the study of MOOCs and the lack of rational reflection on the study of MOOCs.
\end{abstract}

\section{Keywords}

MOOCs, Literature Review, Teaching Mode

\section{Introduction}

"MOOCs" (Massive Open Online Courses), which combines the quality education resources of top universities at home and abroad, are open to the public, provide teaching resources, provide teaching guidance, and conduct interactive exchanges. The "MOOCs" teaching mode is different from the traditional teacher-centered, book-centered and classroom-centered teaching mode, which is the product of "Internet + education". The term "MOOCs" was first proposed by Canadian scholars Dave Cormier and Bryan Alexander in 2008. In 2012, the large-scale promotion of "MOOCs" attracted worldwide attention. This year was called "The Year of MOOC" by The New York Times. Up to now, the world's major platforms, such as Coursera, Udacity, Edx, FutureLearn, Canvas Network, etc., have brought together 8401 courses from world-renowned universities, such 
as Stanford University, Manchester University, Harvard University, MIT, and Hong Kong University. ${ }^{1}$ Domestically, a variety of MOOCs platforms, such as "Chinese University MOOC", "School Online" and "Yi Xian Meng Ke", have been created. With the rise and the large-scale development of MOOCs, the teaching mode of MOOCs as a new thing has changed the traditional teaching ecology to a large extent and has become a new way for people to learn knowledge. This emerging phenomenon has aroused great attention from experts and scholars in related fields and has formed a large number of valuable research results. Therefore, this paper summarizes the characteristics, influences and development prospects of the MOOCs teaching mode by retrieving and system finishing the journals, monographs of the MOOCs research in recent years. In order to deeply understand the research on the teaching mode of MOOCs, I hope to help the research of relevant scholars.

\section{The Development and Characteristics of the Teaching Mode of MOOCs}

The teaching mode of MOOCs can be regarded as a new type of knowledge communication mode. This knowledge communication mode has obvious characteristics different from the traditional teaching mode. It is precisely because of these new features that the MOOCs have formed self-development advantage under the background of the information age. It has become a unique teaching mode that has changed the way people learn. Li Qing and Wang Tao summed up the five characteristics of the MOOC, that is, the MOOCs is a curriculum model, an open form of education, a giant course with a large number of participants, with openness and individuality. And MOOCs is a generative course [1]. Chai Yue, Wu Wenying and Yang Liansheng believe that MOOC combines the characteristics of online media, showing the characteristics of large-scale, open, online and course integrity, but unlike the general online course. The MOOC showed the connotation characteristics of the audience as the knowledge dissemination center, and formed a new knowledge dissemination model with platform as the environment, students as the center and interactive, which changed the knowledge communication pattern and affected the overall development trend of knowledge dissemination [2]. Viktorija Florjančič (2016) conducted a survey of how the EU (including the UK) people learn, and the results show that e-learning has become a learning channel for more people. For example, more than $70 \%$ of the 195 corporate learning and HR professionals at Future Workplace have integrated MOOCs into their learning programs [3].

Through the existing cases and research, it can be considered that the MOOCs has the following characteristics: the MOOCs is characterized by large-scale, open, free, personalized, geographically independent, networked teaching, etc. "Massive", "openness" and "online learning" are considered to be the most obvious features of MOOCs.

${ }^{1}$ Data Sources: Class Central https://www.class-central.com/. 


\subsection{The Characteristics of the "Massive" Teaching Mode of MOOC}

Cai Wenzhao and Wang Qiong believe that the "Massive" of MOOCs is expressed in the number of learners. It is different from the traditional courses of dozens or hundreds of learners. A MOOCs course often has tens of thousands of listeners [4]. Yu Zhongning made a statistic. The four courses of Peking University were officially launched on the edX platform which is the first global sharing courses on the mainland. Only one day after the start of the course, there are more than 20,000 people online in the world [5]. Wang Manqi (2015) believes that the "Massive" characteristics of MOOCs are summarized in four aspects, namely, the large scale of student groups, platforms, teacher teams and curriculum scale. Taking the teacher team as an example, making a MOOCs is a systematic project involving courseware, teaching, video production, etc., which requires a large team of teachers to complete effectively [6].

\subsection{The Characteristics of the "Openness" Teaching Mode of MOOCs}

Cormier and Siemens interpret "openness" as that once these learners are involved in our courses, they will enter our research facility, join our research and discussion, and contribute to exploring the unknown in a particular field of study [7]. Wang Manqi expounded the openness of the MOOCs, that is, the "MOOCs" is fully open to the educated group, and the "MOOCs" course is tested and supervised by the whole society, the same industry and various educated people. "MOOCs" also has a more open teaching form and learning form, and the openness of the "MOOCs" teaching mode lies in the opening of the educational concept [6]. Li Qing and Wang Tao believe that "MOOCs" promote global resource sharing and curriculum opening, and become a new choice for learners in the global network era [1]. In view of the "Openness" of MOOCs, some scholars have also reflected on it. Some scholars have also reflected on the openness of MOOCs. The openness of "MOOCs" has also led to different learners' significant differences in curriculum participation and curriculum contributions, the level of participation of learners can be divided into potential learners, more active learners and very active learners [8]. Johh Daniel believes that although the open nature of "MOOCs" makes this teaching model unique, openness cannot be considered as a driving force for the sustainable development of "MOOCs". This motivation should be considered as a substantial innovation in the curriculum model [9].

\subsection{The Characteristics of the "Online-Learning" Teaching Mode of MOOCs}

Zhang Yunjiao summarizes the characteristics of MOOCs' "online learning". "Online learning" is a two-way interaction between teachers and teachers, students and students, which means economical savings and convenience, real-time monitoring of learning data [10]. Chen Xiaogeng and Wang Dingming believe that big data analysis is an important performance of the "online learning" fea- 
ture of MOOC. For example, MIT invested $\$ 200,000$ in data characterization and mining of the "Circuit and Electronics" course to explore and discover more suitable online teaching content, learning objects and teaching methods [11]. Liu Yang and Huang Zhenzhong think that compared with the traditional teaching mode, the "online teaching" characteristics of MOOCs have formed a huge subversive advantage, which is of great significance to the improvement of the quality and level of China's higher education [12].

\section{The Interactive Mode of "Teaching" And "Learning" in the Teaching Mode of MOOCs}

The biggest difference between the MOOCs teaching mode and the traditional teaching mode is reflected in the change of teaching methods. Under the application of new technology, the MOOCs teaching mode breaks the limitations of time, space and audience in the traditional teaching mode, and forms a new interactive mode of "teaching" and "learning". In this mode, teaching design, teaching behavior, learning behavior and teaching evaluation all present new features and requirements.

\subsection{The Interaction between "Teaching" and "Learning" in the Teaching Mode of MOOCs}

Sun Nian and Zhang Youzhen in the study of the operation mode of the MOOCs, they believe that the interaction between "teaching" and "learning" in the MOOCs is manifested in three ways: teacher evaluation, student mutual evaluation and student feedback. It is conducted through three channels: mail, public forums and course discussion areas. This approach has transformed the traditional teaching model that is teacher-led and students are passively accepted. Students become the initiative of learning, but due to the lack of face-to-face communication, the guiding role of teachers is neglected, and students lose the opportunity of actual communication [13]. Li Qing and Wang Tao from the perspective of Unicomism, that four principles are presented in MOOCs teaching: 1) the principle of convergence. A vast amount of knowledge is gathered in the teaching platform for learners to personalize their choices. 2) The principle of mixing. The teacher's curriculum resources are integrated with the learner's own learning resources. 3) The principle of transfer. MOOCs teaching encourages students to innovate based on existing courses. 4) Promote the principle of sharing. MOOCs teaching can promote learners from all regions to share knowledge, and teachers can easily re-aggregate some valuable knowledge [1]. Li Huajuan in the study of the interaction between "teaching" and "learning", highlights the lack of emotional teaching in the teaching of MOOC, which is manifested as 1) the imbalance of knowledge transfer and emotional care in the teaching design concept. 2) In the design process, MOOCs pays more attention to the growth of students' knowledge and ability, but neglects the cultivation of learners' personality and spirit, that is, it is difficult to realize the emotional exchanges between teachers and students. 3) Lack of interaction between real 
people, it is difficult to stimulate the enthusiasm of teachers, but also reduce the enthusiasm of students to learn independently. This lack of MOOCs directly affects its development in the future [14].

\subsection{Teaching Design of MOOCs}

In the teaching design, MOOCs should fully consider the characteristics of the MOOCs and the interaction between teachers and students under the new teaching mode. Mike Wald based on a survey of 30 MOOCs on seven different platforms, raised the issues that educators should consider when designing the MOOCs curriculum. 1) The MOOCs should be designed to attract learners to participate and complete the course in the event that the participants have less time and participants withdraw. 2) The design of MOOCs should consider how to compress the content of the course in the traditional classroom for a short period of time to adapt to the teaching form of MOOCs. 3) The design of MOOCs may also be faced with the challenge of copyright rules. In general, there is a big difference between MOOCs and traditional teaching. The characteristics of time and space restrictions in MOOCs also make MOOCs more focused on how to provide attractive and valuable knowledge to learners [15].

\subsection{Teaching Behavior and Learning Behavior in MOOCs Teaching}

\subsubsection{Teacher's Teaching Behavior}

The teaching mode of MOOCs affects the teaching behavior of teachers. Teachers need to change the teaching ideas and teaching methods in the traditional teaching mode to adapt to the MOOCs teaching mode. Fang Xu and Yang Gaixue constructed a factor model for the intention of teaching behavior of college teachers' MOOCs, and studied the factors affecting teacher behavior in MOOCs teaching. The study found that external support perception and perceived ease of use will have an impact on teacher behavior. For example, the capital needs of the teaching of the MOOC and the ability of the teachers themselves, and the influence of the former is more significant. Therefore, universities should increase their support and guidance to teachers in the process of developing MOOCs [16]. Tracy L. Durksen, Man-Wai Chu, and Zaheen F. Ahmad believe that teachers should pay more attention to the motivational research of learners. Teachers face the challenge of how to construct a teacher-student interaction with learners when facing many learners [17]. Gilly Salmon, Janet Gregory, Kulari Lokuge Dona and Bella Ross pointed out that as a teacher in the MOOCs teaching mode, they should be good at finding collaborators, and use the MOOCs platform to form relevant teaching teams to share teaching ideas and teaching experience. In the development process, the MOOCs platform can guide the teaching students to generate the above teaching ideas and learning methods through the online learning design and the corresponding technical aspects [18].

\subsubsection{Student's Learning Behavior}

The teaching mode of MOOCs puts forward new requirements for students' 
learning behaviors. The initiative of the students is the key to the learners' study of the MOOCs. At the same time, the MOOCs should also be adapted to the needs of students' learning behaviors. Zhang Qianfan, Wang Chengyu and Zhang Yajun explored the factors influencing the college students' intent to learn the MOOCs, and concluded that the students' learning behavior is influenced by the quality of the MOOCs teaching resources, the course design of the MOOCs, the guidance of the teaching staff, and the social resources inputting. We need to improve on these aspects so that students can show more intense learning behaviors [19]. Gilly Salmon, Ekaterina Pechenkina, Anne-Marie Chase and Bella Ross argue that the motivations and expectations of MOOCs' learners directly influence their participation in the MOOCs' curriculum. The exit that occurs during the teaching of the MOOCs is now in many cases because the learner's expectations are too high. MOOCs learners' desire for new knowledge and personal development goals will increase their participation if they are implemented in the MOOCs. In addition, students who are flexible enough to adapt to MOOCs teaching, that is, they are active learners, can complete the learning of MOOCs more successfully [20]. Gilly Salmon, Janet Gregory, Kulari Lokuge Dona and Bella Ross believe that because of the characteristics of the MOOCs teaching model, for the learners, especially the non-active learners, the successful use of the MOOCs platform requires self-discipline and self-motivation. And they have to take the initiative to adapt to the teaching language and environment of MOOCs [21].

\subsection{Teaching Evaluation of MOOCs}

In the study of the interactive relationship between the teaching and learning of the MOOCs teaching mode, it is very important to evaluate the teaching of the MOOCs. The research on the evaluation of MOOC teaching mainly involves two aspects: One is the comprehensive evaluation of the teaching design, content and effect of the MOOC, which is more about the provider of MOOCs. They should be promoted to provide quality MOOC teaching resources. The second is the evaluation of the learner's learning process, and feedback on the learner's learning through specific evaluation methods.

\subsubsection{Teaching Evaluation of the MOOCs}

With the rapid development of the teaching mode of MOOCs, a large number of MOOCs resources have been generated. However, these resources are mixed. Therefore, it is necessary to formulate a scientific evaluation system for MOOCs to evaluate the MOOCs, to impose scientific management on MOOCs, and to improve MOOCs' quality. Qiu Junping and Ou Yufang designed the quality evaluation index system of the MOOCs according to the characteristics of the MOOCs, and constructed five first-level indicators including the teaching team, teaching content, teaching effect, teaching resources and teaching technology. And 14 levels of secondary indicators such as MOOCs providing organization, teaching content design, richness of teaching resources, achievement of learning 
objectives, user interface layout, and design specific evaluation content for each secondary index, and then based on the analytic hierarchy process (AHP) designed the weights of all parties, and finally scored the MOOC by fuzzy analysis [22].

\subsubsection{Learning Assessment of Learners in MOOCs}

As a teaching mode, MOOCs is naturally to evaluate the learner's learning situation. However, many learners in the MOOCs have made it an important topic for the teaching staff or related technical personnel to evaluate the learners in the MOOCs teaching mode. With the development of the MOOCs, in addition to the traditional teacher evaluation, self-evaluation, especially peer evaluation, has become an important evaluation method and has considerable development prospects. Xu Tao proposed five peer-scoring models under the teaching mode of MOOCs from the perspective of teaching method and educational technology application, namely peer score model, expert play peer evaluation model, social network peer evaluation model, cross-cultural peer review model and critical peer review model. At the same time, they pointed out that in the design of peer review, it is necessary to pay attention to the consistency of job design and evaluation criteria, the anxiety of learners on peer review, the clarity of evaluation criteria, whether the evaluation criteria are suitable for learners and the fairness of evaluation criteria. These are all significant for the success of peer review [23].

\section{The Influence of MOOCs Teaching Mode on Traditional Teaching}

The development of the teaching mode of MOOCs has injected new methods and ideas into the reform of the traditional teaching mode. For the traditional teaching mode, the full use of modern information technology, the effective use of traditional educational resources, the combination of teacher-led teaching and student independent learning has become the goal of the traditional teaching model. At the same time, the teaching mode of the "MOOCs + flipping classroom" has become an important direction for scholars to study.

\subsection{Reform of Traditional Teaching Model under the Influence of MOOCs Teaching Mode}

\subsubsection{Classroom Teaching Reform under the Influence of MOOCs}

Li Puhua, Xue Hongli and Zhao Yutao pointed out the direction of classroom teaching reform in the era of MOOCs from the perspective of multi-subjects, namely learners, teachers and managers: 1) From the perspective of learners, the concept of autonomous learning should be formed to realize the change of students from "learners" to "mastery" of knowledge. 2) From the perspective of teachers, teachers should use the characteristics of quick update and flexible form MOOCs to reconstruct and update the content of the original lesson. At the same time, teachers should also establish a multi-dimensional evaluation system that includes students' learning experiences, curriculum sharing, and 
teaching effects. 3) From the perspective of managers, we should promote the reform of traditional teaching concepts, techniques and models, and improve the level of curriculum construction and the quality of education and teaching [24]. $\mathrm{Wu}$ Weizhong, Guan Xiaohui and Qu Chaoyang believe that deepening the reform of classroom teaching, MOOCs may be a good prescription, but the key is to deal with the relationship between "online" and "offline". Online learning classes, students can grasp the key points and difficulties of the course in advance, through the quiz and forum, teachers can also quickly understand the learning situation of students. Discussion and improvement under the line, teachers and students concentrate on solving key problems in the classroom, and provide individualized guidance for students to achieve teaching efficiency and improve teaching quality [25]. Song Zhuanmao proposed the reform method of college teaching methods based on MOOCs from the perspective of integrated thinking, which enlightened and cultivated integrated teaching thinking, which enabled the educators to make full use of modern information network technology to provide technical support for the reform of teaching methods. At the same time, teachers can also accurately understand the teaching world under the new resource conditions. This approach can also create and promote an integrated teaching resource analysis framework, break through existing teaching models and methods, create new order, paradigm and culture, achieve seamless integration between online and offline teaching, in-class and extra-curricular teaching, avoid misuse and abuse of information technology, ensure effective teaching and the operation of colleges and universities [26].

\subsubsection{The Influence of MOOC on the Teaching Reform of Specific Courses} Chen Hao believes that today's ideological and political theory courses in colleges and universities are faced with the syllabus of ignoring the syllabus, neglecting the individual needs of learners, or unilaterally highlighting individual functions, abandoning social requirements and principles. The "MOOCs" platform can achieve social functions and meet the individual development needs of students through its own interactive performance [27]. Wang Shouhong, Liu Jinling and Fu Wenping attempted to construct a content-based college English ESP teaching model (MCE) with the help of modern information technology represented by the network "MOOCs". Under the MCE mode, the "online" and "offline" teachings are integrated. "Online" is arranged for students to take part in self-learning tasks in the class. "Offline" combines other learning materials with students' self-learning tasks and conducts various forms of classroom interaction learning with students. Based on this, the scientific nature of this model is also verified through a small range of empirical studies [28].

\subsection{The Teaching Mode of "MOOCs + Flipping Classroom"}

Chen Yuxi believes that the teaching mode of "MOOCs + flipping classroom" is a teaching mode of pre-learning, with the characteristics of media diversification, dynamic process visualization, learning feedback, simplification of process 
evaluation and resource globalization. At the same time, the teaching mode of "MOOCs+ flip classroom" has formed its own unique advantages in the "online" and "offline" teaching. 1) The "online" MOOCs are presented in the form of micro-courses. The teacher builds a knowledge map for the students, so that the students can "accord to the drawings according to the requirements of the curriculum design"; design "advanced assignments". After students understand what they have learned at this stage, they can enter the next stage of learning, which is conducive to consolidating every step of the student's learning. 2) The "offline" flipping classroom has formed four types of consolidation and strengthening, systematic combing, deepening and innovating in the teaching activities of the post-learning teaching. In the flipping classroom, because the students have completed the "online" micro-learning, based on the grasp of the basic knowledge, more inquiry activities can be carried out in the classroom, which is conducive to stimulating students' creativity [29]. Tian Aili conducted a survey of more than 100 schools in 20 cities in China that are implementing MOOCs and flip classroom teaching practice projects to in order to in-depth understanding of the practical results of the "MOOCs + flipping classroom" through classroom observation, questionnaire survey and in-depth interviews. According to the survey results, for students, the teaching mode of the "MOOCs + flipping classroom" stimulates students' interest in learning, cultivates students' autonomy and cooperation ability, and promotes students' thinking ability, practical ability and academic achievement. For teachers, it enhances teachers' academic quality, information technology literacy and classroom teaching management. skills. However, this model still faces the problem that teachers' teaching burden is aggravated, teaching concepts are difficult to change at one time, students lack self-learning, teaching management, teaching evaluation and other related systems are imperfect, and they are restricted by teaching resources and funds [30].

\section{Advantages, Disadvantages and Improvement Strategies of MOOCs Teaching Mode}

\subsection{Advantages and Disadvantages of MOOCs Teaching Mode}

The emergence of the teaching mode of the MOOC changed the interaction between "teaching" and "learning" in traditional classroom teaching, and profoundly affected the reform of the traditional teaching model. With the development of science and technology in the information age, the MOOCs teaching mode has formed the advantages that traditional teaching does not have. However, it is because of the large-scale, open and personalized characteristics of the MOOCs, there has arisen a series of problems such as high production costs, high student dropout rates, and lack of emotional communication.

Li Qing and Wang Tao believe that the MOOCs teaching mode has advantages such as ease of use, free use, diversification of tools and resources, independent learning and social construction compared with the traditional open curri- 
culum. Among them, social construction is the most prominent feature of the MOOCs teaching mode, that is, learners from all walks of life generate new knowledge through the collision of thinking. However, there are certain shortcomings in the MOOCs. For example, the construction of a MOOCs course must have a solid knowledge base, excellent organization and coordination ability for the initiators. The multi-discipline and dispersal of the learners make it difficult to achieve harmony and coordination. The differences of knowledge background and cultural also cause communication difficulties [1]. Wang Yingjie, Feng Ce and Nie Wei believe that MOOCs has its own inherent multiple contradictions. The contradiction between the openness of the teaching mode of the MOOCs and the closedness of the target audience of the MOOCs, that is, the setting of each course is directed to a certain degree of learning group, but this group is limited. This feature is in conflict with the characteristics of large-scale openness of the class. The inherent contradiction between the self-organization of the MOOCs and the organization of group learning, that is, the students spontaneously choose courses and classes, and the teachers spontaneously open the MOOCs courses. This makes the teaching organization and curriculum system of MOOCs fall into an unorganized state [31]. Zhu Qingfeng also believes that the inherent contradiction of the MOOCs has led to the emergence of the crisis of the MOOCs. The form of contradiction is mainly reflected in the contradiction between the self-organization of the MOOCs and its organization, the contradiction between the high autonomy and high exit rate of the MOOCs, the contradiction between the utilitarian and moral ethical conflicts of the MOOCs and the contradiction between cultural and national contradiction of the MOOCs [32]. Wang Haibo summarized the problems encountered in the development of MOOCs teaching mode from different angles in foreign countries. 1) In the design of the MOOCs course, the design ideas of some MOOCs still remain in the traditional teaching mode, and students are still regarded as passive recipients. In the evaluation process, the MOOCs course is composed of choice, judgment and simple question and answer. It cannot cultivate students' critical thinking and problem-solving ability. In the teaching process, the interaction between the teacher and the learner is also very limited. 2) In terms of MOOC learners, the learners of MOOCs are mostly adults with university degrees, and there is still a very high dropout rate. 3) In the colleges and professors who make MOOCs classes, the high economic and time costs in the production of MOOCs make the professors spend a lot of time and energy. And it is also in dispute as to whether the MOOCs can improve the quality of school teaching and promote teaching research. Many colleges and universities have begun to resist the MOOCs. 4) In the MOOCs company, whether it is profitable or not has become a key issue in the development of MOOCs [33].

\subsection{The Improvement Strategy of MO0Cs Teaching Mode}

Aiming at the problems existing in the teaching mode of MOOCs, some scholars 
have also strengthened the discussion on the improvement strategy of MOOCs teaching mode. Feng Yonghua and Liu Zhijun analyzed from many angles that the teaching of MOOCs should achieve the following in the development process. 1) Establish a new concept of MOOCs culture. The instructor should recognize that the open curriculum culture of the MOOCs provides the possibility of the implementation and generation of the dynamic curriculum, and the non-linear course of operation and the diversity of course evaluations reflect the curriculum culture centered on "learning". 2) Building a new way of organizing courses. Teachers need to change their working methods and roles, and they are truly the guides of knowledge. At the same time, teachers need to explore the teaching methods of MOOCs. 3) Practice the "relationship" of the MOOCs. Under the relationship thinking, the elements of the MOOCs should form an organic interaction. 4) Construct an external support condition framework for the MOOCs. Government management departments actively guide, platform operators provide flexible and professional technical services and form a quality MOOCs development team [34]. Zhang Ziyi and Song Shuhong proposed the improvement strategy of the MOOCs teaching mode from the audience group, teaching group and social perspective of MOOCs teaching. 1) From the perspective of the audience group, it is necessary to innovate the traditional learning methods and reasonable fees to mobilize the students' enthusiasm for learning; 2) From the perspective of the teaching group, it is necessary to recognize that the learners of the MOOCs are not limited to the students, and the lecturers need to change the teaching methods. 3) From the social point of view, it should be more in the humanized design of the content and interface of the MOOCs [35]. Yuan Luqi proposed an improvement strategy from the teaching management of universities. 1) Improve the teaching management system of the MOOCs; 2) Pay attention to the management of the teaching objectives of the MOOCs; 3 ) Strengthen the construction and management of the MOOCs curriculum; 4) Improve the management and incentive mechanism for MOOCs teachers; 5) Carry out the "student-centered" teaching management of MOOCs [36].

\section{Conclusion}

Starting from the teaching mode of the MOOCs, the researchers have done a lot of research on the development and characteristics of the teaching mode of the MOOCs, the interactive relationship between "teaching" and "learning", the influence of the teaching mode of the MOOCs on the traditional teaching mode, the advantages and disadvantages of the teaching mode of the MOOCs, and the improvement strategy of the teaching mode of the MOOCs. However, it should also be found that there are still some shortcomings in the study of MOOCs. There are three main aspects: 1) Insufficient research on the improvement strategy of MOOCs. In the design of the improvement strategy of the MOOCs, there is a lack of operability exploration of the design content, making it difficult to implement it; and most of the design solutions are merely supplementing the 
existing problems and lacking innovation. 2) There is no empirical analysis of the study of MOOCs. In the study of the MOOCs, it is more based on theory or only from some characteristics or phenomena of MOOCs. There is a lack of in-depth analysis of a course or a series of courses, lack of scientific quantitative analysis, making the research persuasive insufficient. 3) There is no rational reflection on the study of MOOCs. Most of the research is in a frenzied pursuit of the MOOC, and it lacks doubts about the teaching mode of MOOCs. Therefore, the researcher may be able to develop and innovate the research on the teaching mode of the MOOCs in the above three questions, and this research summary on the teaching mode of the MOOCs also hopes to provide some help to the researchers.

\section{Conflicts of Interest}

The author declares no conflicts of interest regarding the publication of this paper.

\section{References}

[1] Li, Q. and Wang, T. (2012) MOOCs: A Giant Open Course Model Based on Connectivism. Distance Education in China.

[2] Chai, Y., Wu, W.Y. and Yang, L.S. (2015) Study on the Construction of New Knowledge Communication Model in MOOCs. Journalism Education Research.

[3] Uden, L., Liberona, D. and Feldmann, B. (2016) Learning Technology for Education in Cloud-The Changing Face of Education. Springer International Publishing, Berlin. https://doi.org/10.1007/978-3-319-42147-6

[4] Cai, W.X. and Wang, Q. (2013) 2012: The First Year of MOOCs. China Education Network.

[5] Yu, Z.N. (2013) "Mu Class" Strikes, Is the Traditional University Still Safe? Workers Daily.

[6] Wang, M.Q. (2015) The Comments and Suggestions on MOOCs Teaching Mode. Inner Mongolia Normal University.

[7] Cormier, S. (2010) Through the Open Door: Open Courses as Research, Learning and Engagement. Educause Review.

[8] mobiMOOC. (2011) Learning Actions. http://mobimooc.wikispaces.com/Learning+actions+-+plan+your+learning

[9] Daniel, J. (2012) Making Sense of MOOCs: Musings in a Maze of Myth. Paradox and Possibility.

[10] Zhang, Y.J. (2015) The Compared Research of MOOCs and the Traditional Teaching Method. Inner Mongolia Normal University.

[11] Chen, X.G. and Wang, D.M. (2013) Analysis of the Development Process and Main Characteristics of MOOC. Modern Educational Technology.

[12] Liu, Y., Huang, Z.Z., Zhang, Y. and Li, M.L. (2013) A Survey Report on the Participation of Chinese MOOCs Learners. Tsinghua University Education Research.

[13] Sun, N. and Zhang, Y.Z. (2015) The Operating Mode and Teaching Interaction Mechanism of MOOCs. Higher Education Development and Evaluation.

[14] Li, H.J. (2016) The Dilemma and Outlet of Emotional Education in College MOOCs 
Teaching. Education and Vocation.

[15] Wald, M. (2016) Computers Helping People with Special Needs. Springer International Publishing, Switzerland.

[16] Fang, X. and Yang, G. (2016) Research on the Factors of College Teachers' Behavior Intention for MOOC Teaching. Open Education Research.

[17] Durksen, T.L., Chu, M.-W., Ahmad, Z.F., Radil, A.I. and Daniels, L.M. (2016) Motivation in a MOOC: A Probabilistic Analysis of Online Learners' Basic Psychological Needs. Social Psychology of Education, 19, 241-260.

https://doi.org/10.1007/s11218-015-9331-9

[18] Salmon, G., Gregory, J., Dona, K.L. and Ross, B. (2016) Experiential Online Development for Educators: The Example of the Carpe Diem MOOCs. British Journal of Educational Technology, 46, 542-556.

[19] Zhang, Q., Wang, C. and Zhang, Y. (2015) An Empirical Study on the Influencing Factors of College Students' Intention to Study in MOOCs. Higher Education Exploration.

[20] Salmon, G., Pechenkina, E., Chase, A.-M. and Ross, B. (2016) Designing Massive Open Online Courses to Take Account of Participant Motivations and Expectations. British Journal of Educational Technology, 48, 1284-1294.

[21] García, B.J., Tenorio, G.C. and Ramírez, M.S. (2015) Self-Motivation Challenges for Student Involvement in the Open Educational Movement with MOOC. International Journal of Educational Technology in Higher Education, 12, 91-103.

[22] Qiu, J.P. and Ou, Y.F. (2015) Research on the Construction and Application of the Quality Evaluation Index System of MOOCs. Higher Education Development and Evaluation.

[23] Xu, T. (2015) Design of Peer Assessment in MOOCs. Open Education Research.

[24] Li, P.H., Xue, H.L. and Zhao, Y.T. (2016) Analysis of Blended Learning Based on MOOCs from the Multi-Agent Perspective. Journal of Education Management, 6, $88-92,124$.

[25] Wu, W.Z., Guan, X.H. and Qu, Z.Y. (2015) Discussion on Higher Education Reform Initiated by MOOCs Wave. Journal of Northeast Normal University, 2, 190-194.

[26] Song, Z.M. (2014) How to Promote Teaching Method Reform in Higher Education Institutions through MOOCs. Fudan Education Forum.

[27] Chen, H. (2014) The Enlightenment of "MOOCs" on the Teaching Reform of Ideological and Political Theory Courses in Colleges and Universities. Ideological \& Theoretical Education.

[28] Wang, S.H., Liu, J.L. and Fu, W.P. (2015) A Study on the Content-Based College ESP Instruction under the Background of MOOCs Era. China Educational Technology.

[29] Chen, Y.K. (2014) Research on Combined Model of MOOCs and Flipped Classroom in Primary and Secondary Schools. Curriculum, Teaching Material and Method, No. 10, 10-17 + 33 .

[30] Tian, A.L. (2015) An Empirical Study of the MOOCs plus Flipped Classroom Learing Model. Open Education Research.

[31] Wang, Y.J., Feng, C. and Nie, Y. (2015) Situation Analysis of MOOCs in China Higher Education and Countermeasure. China Educational Technology.

[32] Zhu, Q.F. (2014) Difficult and Rational Choices of the MOOCs Development in 
China's Higher Education. Research in Education Development.

[33] Wang, H.B. (2015) Analysis of the Problems Existing in the Development of Current MOOCs in Foreign Countries. Fudan Education Forum.

[34] Feng, Y.H. and Liu, Z.J. (2017) The Problems, Causes and Improving Paths of MOOCs Development-Based on the Investigation into Teachers of Colleges Offering MOOCs. Modern Distance Education Research.

[35] Zhang, Z.Y. and Song, S.H. (2016) Education Production Relationship Problems of Credit Accumulation and Transformation in Higher Vocational Education. Modern Education Science (Primary School Headmaster).

[36] Yuan, L. (2018) The Study of MOOCs Teaching Management in University. Hunan University, Changsha. 